This document was prepared in conjunction with work accomplished under Contract No. DE-AC09-96SR18500 with the U. S. Department of Energy.

\title{
DISCLAIMER
}

This report was prepared as an account of work sponsored by an agency of the United States Government. Neither the United States Government nor any agency thereof, nor any of their employees, nor any of their contractors, subcontractors or their employees, makes any warranty, express or implied, or assumes any legal liability or responsibility for the accuracy, completeness, or any third party's use or the results of such use of any information, apparatus, product, or process disclosed, or represents that its use would not infringe privately owned rights. Reference herein to any specific commercial product, process, or service by trade name, trademark, manufacturer, or otherwise, does not necessarily constitute or imply its endorsement, recommendation, or favoring by the United States Government or any agency thereof or its contractors or subcontractors. The views and opinions of authors expressed herein do not necessarily state or reflect those of the United States Government or any agency thereof. 


\section{RHEOLOGICAL MODIFIER TESTING WITH DWPF PROCESS SLURRIES}

M. E. Stone

A. R. Marinik

D. M. Marsh

February 2004

Immoblization Technology Section Savannah River Technology Center Aiken, SC 29808 
This page was intentionally left blank 
WSRC-TR-2004-00082

Revision 0

Key Words: DWPF, Rheology, SRAT, SME, Dispersant

Retention: Permanent

\section{RHEOLOGICAL MODIFIER TESTING WITH DWPF PROCESS SLURRIES}

M. E. Stone

A. R. Marinik

D. M. Marsh

February 2004

Immoblization Technology Section Savannah River Technology Center Aiken, SC 29808 
This page was intentionally left blank 


\section{EXECUTIVE SUMMARY}

Rheological modification agents were tested on simulated SRAT and SME products to determine if a suitable agent could be found for the DWPF process slurries. The agents tested were dispersants that lower the rheological properties of slurries by preventing agglomerization. Dolapix CE64, an ethylene glycol, and Disperse-Ayd W28, a polyacrylate, were the most effective dispersants tested.

Further evaluation and testing should be performed on Dolapix CE64 and Disperse-Ayd W28 to determine if implementation is possible in DWPF. The initial phase of future work will include optimization of the rheology modifier by the Illinois Institute of Technology (IIT) and development of a maximum concentration limit for the rheology modifiers. IIT has been commissioned to evaluate the properties of these chemicals to determine if the chemical makeup can be optimized to enhance the properties of these modifiers. An initial concentration limit based upon the DWPF flammability limit and other constraints should be calculated to determine the potential downstream impacts.

Once an optimized rheology modifier has been recommended by IIT, the second phase of future work concerning rheology modifiers should focus on the following areas:

- Effective $\mathrm{pH}$ range of dispersants

- Effect of boiling on dispersants

- Effect of aging on dispersants

- Impact of dispersants on foaming

- Impact of dispersants on hydrogen generation

- Impact of dispersants on glass redox

- Impact of dispersant components (such as ammonia) on process

It is anticipated that the second phase of this program will start in FY05. 


\section{TABLE OF CONTENTS}

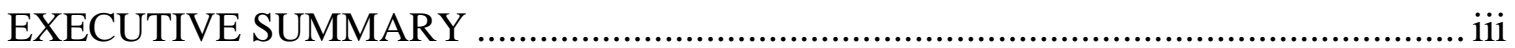

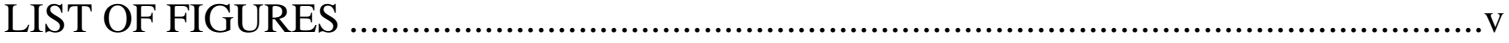

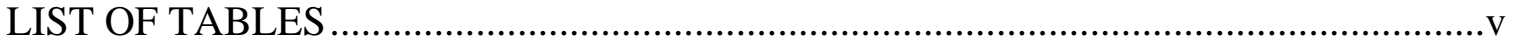

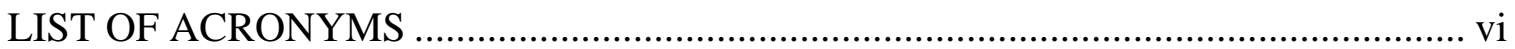

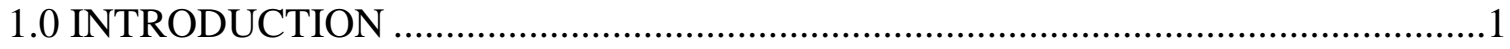

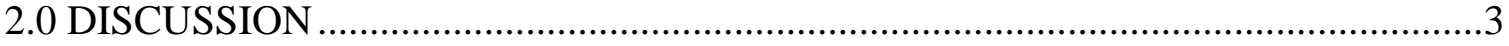

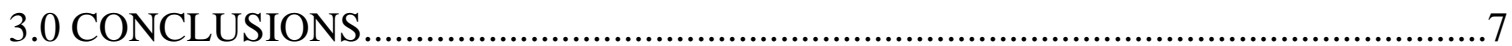

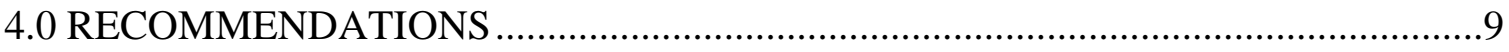

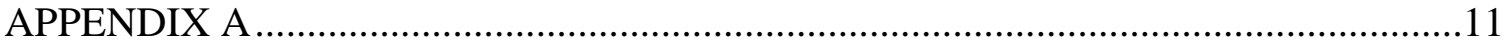




\section{LIST OF FIGURES}

Figure 1. Impact of Water Additions on Yield Stress of SME Product.................................... 5

\section{LIST OF TABLES}

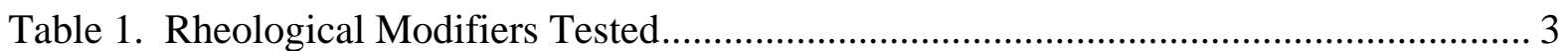

Table 2. Data from Dolapix CE64 and Disperse-Ayd W28 Tests.............................................. 4 


\section{LIST OF ACRONYMS}

DWPF Defense Waste Processing Facility

HLW High Level Waste

IIT Illinois Institute of Technology

MFT Melter Feed Tank

SME Slurry Mix Evaporator

SRAT Sludge Receipt Adjustment Tank 


\subsection{INTRODUCTION}

The Defense Waste Processing Facility (DWPF) at the Savannah River Site (SRS) vitrifies high level radioactive waste (HLW) sludge currently stored at the site. DWPF receives washed HLW sludge in the Sludge Receipt Adjustment Tank (SRAT) on top of the heel from the previous batch. The sludge is then acidified by adding nitric and formic acid. Mercury is then removed from the sludge by reflux boiling through a decanter, and then the sludge is concentrated by evaporation. The concentrated sludge is transferred to the Slurry Mix Evaporator (SME). In the SME, the sludge is combined with the glass formers which are transferred to the vessel as a frit slurry. The mixture is then evaporated to remove water and decrease the heat load on the melter. SME product is transferred to the Melter Feed Tank (MFT) which slowly feeds the slurry to the melter.

Process upsets have occurred in the DWPF as a result of changes in the rheological properties of the process slurries. Reducing the yield stress of the process slurries is expected to minimize process upsets and to potentially allow higher solids loading in the SME product. Higher solids loading would decrease the amount of water the melter would be required to evaporate and could increase the glass production rate.

In commercial industries, dispersion agents are commonly used to decrease the yield stress of slurries. The dispersion agents prevent agglomerization of the particles in the slurry either by affecting the surface charge of the particles or by blocking the interaction between the particles through steric effects. Commercially available dispersants are usually effective in a narrow $\mathrm{pH}$ range and can act as flocculating agents outside the effective range. Many dispersion agents are based on acrylic polymers with variations in polymer chain length and the addition of other, often proprietary, species to the polymer. 
WSRC-TR-2004-00082

Revision 0

This page intentionally left blank. 


\subsection{DISCUSSION}

Scoping studies conducted during the summer of $2001^{1}$ indicated that addition of a rheological modifier could reduce the yield stress and consistency of simulated waste sludges. Based on this preliminary study, additives were tested with DWPF process slurries to determine if the yield stress and consistency could be reduced. A batch of simulated SRAT product produced during FY02 melt rate testing was utilized to perform the tests. SME product was prepared from this SRAT product by addition of dry frit to the SRAT product. A Haake RS600 research grade rheometer was utilized to measure the rheological properties of the samples using the concentric cylinder geometry. Yield stress and consistency were determined from the data using the Bingham plastic model as described by Koopman. ${ }^{2}$

The additives selected for testing are shown in Table 1, along with additive type and manufacturer. Each additive was tested in SRAT and SME product at various concentrations, as shown in Appendix A.

Table 1. Rheological Modifiers Tested

\begin{tabular}{|c|c|c|}
\hline Additive & Type & Manufacturer \\
\hline Sodium Meta-Silicate & Crystalized silicate & Various \\
\hline Sodium Polyphosphate & Phosphate polymer & Various \\
\hline Darvan 7 & Polymethylacrylate, anionic & Vanderbilt Co. Inc. \\
\hline Duramax 3005 & Ammonium Polyacrylate & Rohm and Haas \\
\hline Dolapix CE64 & Proprietary Ethylene Glycol & Zschimmer and Schwartz \\
\hline Disperse-Ayd W22 & Proprietary Polyacrylate & Elementis Specialties \\
\hline Disperse-Ayd W28 & Proprietary Polyacrylate & Elementis Specialties \\
\hline Disperse-Ayd W30 & Proprietary Polyacrylate & Elementis Specialties \\
\hline Disperse-Ayd W39 & Proprietary Polyacrylate & Elementis Specialties \\
\hline Alcosperse 149 & Sodium polyacrylate & Alco Chemical \\
\hline Alcosperse 240 & Proprietary Polyacrylate & Alco Chemical \\
\hline Alcosperse 408 & Proprietary Polyacrylate & Alco Chemical \\
\hline Alcosperse 725 & Proprietary Polyacrylate & Alco Chemical \\
\hline EDA Plan 470 & Proprietary Polyacrylate & Ultra Additives \\
\hline EDA Plan 472 & Proprietary Polyacrylate & Ultra Additives \\
\hline Pomosperse AL36 & Proprietary Polyacrylate & Piedmont Chemical Co. \\
\hline Cyanamer P-35 & Proprietary Polyacrylate & Cytec \\
\hline Cyanamer P-70 & Proprietary Polyacrylate & Cytec \\
\hline Sugar & Glucose & Various \\
\hline
\end{tabular}

${ }^{1}$ E. D. Kay, et al. “Rheological Modifiers for Radioactive Waste Slurries”, WSRC-MS-200300136, July 2003.

${ }^{2}$ D. C. Koopman and D. H. Miller, Production of Simulated Sludge Batch 2 Melter Feed Containing Frit 320 in the $1 / 240^{\text {th }}$ Glass Feed Preparation System, WSRC-TR-2002-00186, Revision 0, April 22, 2002. 
The majority of the additives tested led to increased yield stress and consistency, but several led to significant improvements. The results from the two most effective dispersants are shown in Table 2. The variation in the amount of improvement at a given concentration may be the result of differences in $\mathrm{pH}$ in the slurries tested since the same SRAT and SME products were not utilized for all tests. pH was not measured during these tests, but will be added to the test protocol for any future testing.

Table 2. Data from Dolapix CE64 and Disperse-Ayd W28 Tests

\begin{tabular}{|c|c|c|c|c|c|}
\hline Additive & $\begin{array}{c}\text { Sample } \\
\text { Type }\end{array}$ & $\begin{array}{c}\text { Concentration } \\
\text { of Additive in } \\
\text { Sample }\end{array}$ & $\begin{array}{l}\text { Yield } \\
\text { Stress }\end{array}$ & Consistency & $\begin{array}{c}\% \\
\text { Improvement } \\
\text { in Yield Stress } \\
\text { from Baseline }\end{array}$ \\
\hline & & PPM & $\mathbf{P a}$ & $\mathbf{c P}$ & $\%$ \\
\hline \multirow[t]{2}{*}{ Dolapix CE64 } & SRAT & 747 & 10.8 & 13.3 & 8.6 \\
\hline & SRAT & 1492 & 10.2 & 13.3 & 13 \\
\hline \multirow{12}{*}{$\begin{array}{c}\text { Proprietary } \\
\text { Ethylene Glycol }\end{array}$} & SME & 1621 & 16.4 & 20.1 & -4.1 \\
\hline & SME & 3234 & 13.8 & 18.8 & 13 \\
\hline & SRAT & 3234 & 2.5 & 8.9 & 35 \\
\hline & SRAT & 6436 & 2.3 & 8.6 & 41 \\
\hline & SRAT & 9606 & 2.1 & 8.6 & 46 \\
\hline & SRAT & 12745 & 1.7 & 8.3 & 56 \\
\hline & SRAT & 649 & 10.4 & 14.8 & -1.3 \\
\hline & SRAT & 1621 & 9.2 & 14.3 & 10 \\
\hline & SRAT & 3234 & 8.0 & 14.6 & 22 \\
\hline & SME & 649 & 9.70 & 0.023 & 7.6 \\
\hline & SME & 1621 & 9.66 & 0.023 & 8.0 \\
\hline & SME & 3234 & 8.24 & 0.019 & 22 \\
\hline \multirow{3}{*}{$\begin{array}{l}\text { Disperse-Ayd } \\
\text { W28 }\end{array}$} & SRAT & 2289 & 1.8 & 9.0 & 52 \\
\hline & SRAT & 4554 & 1.5 & 5.2 & 62 \\
\hline & SRAT & 6798 & 0.82 & 7.5 & 79 \\
\hline \multirow{8}{*}{$\begin{array}{l}\text { Proprietary } \\
\text { Polyacrylate }\end{array}$} & SRAT & 9020 & 0.60 & 7.6 & 85 \\
\hline & & & & & \\
\hline & SRAT & 460 & 9.5 & 12.4 & 6.8 \\
\hline & SRAT & 1147 & 9.6 & 10.2 & 6.7 \\
\hline & SRAT & 2289 & 10.4 & 8.1 & -1.4 \\
\hline & SME & 460 & 10.8 & 20 & -3.1 \\
\hline & SME & 1147 & 8.6 & 17 & 18 \\
\hline & SME & 2289 & 6.1 & 15 & 42 \\
\hline
\end{tabular}


The impact of water additions on the SME products was tested to compare to the improvement in yield stress provided by the Dolapix CE64 and Disperse-Ayd W28. Water additions reduced the yield stress, but required much larger addition amounts than the dispersant. For a reduction of $20 \%$ in yield stress, the amount of dispersant was less than $1 / 10^{\text {th }}$ the amount of water that would be required. The impact of water is shown in Figure 1.

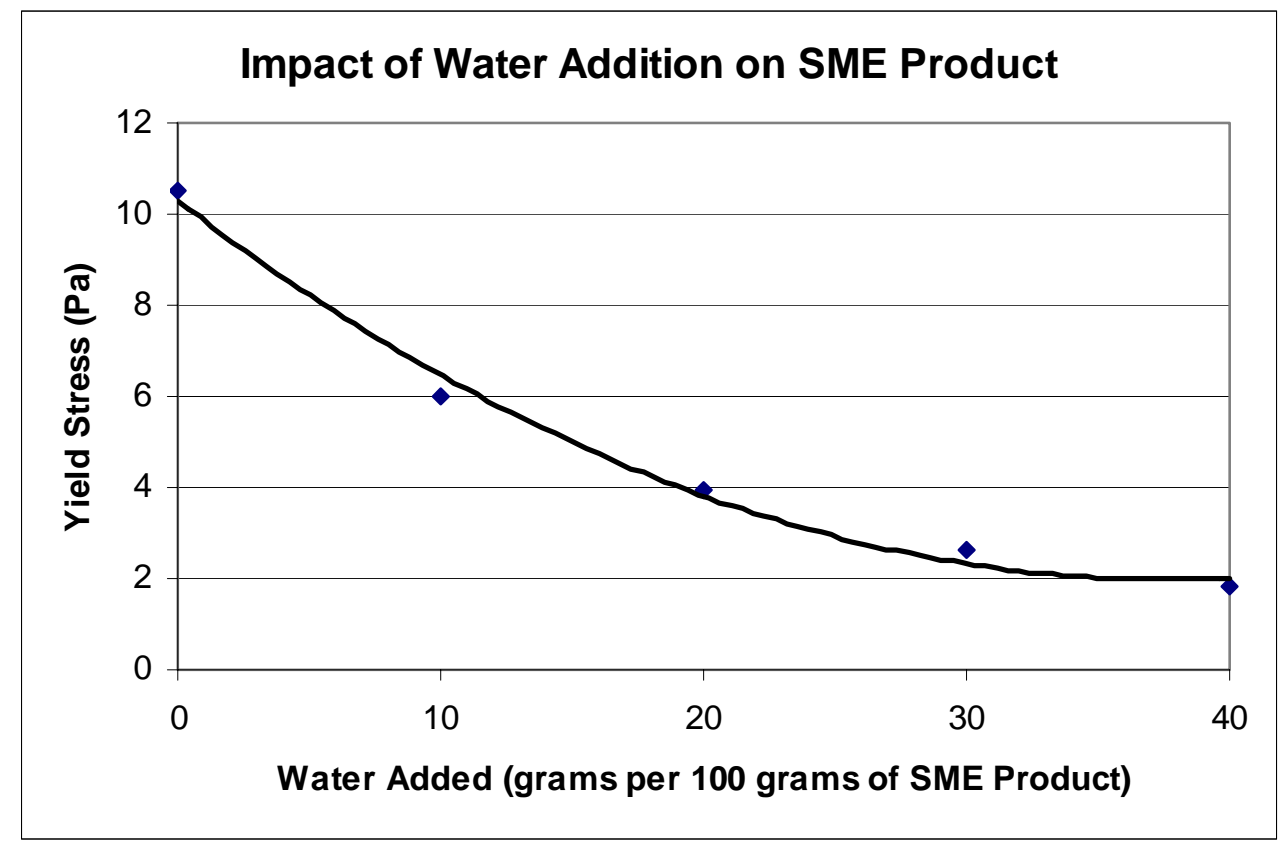

Figure 1. Impact of Water Additions on Yield Stress of SME Product 
WSRC-TR-2004-00082

Revision 0

This page intentionally left blank. 


\subsection{CONCLUSIONS}

Dolapix CE64 and Disperse-Ayd W28 were the most effective dispersants tested at reducing the yield stress of the SRAT and SME products tested. The effectiveness of the additives was likely impacted by the $\mathrm{pH}$ of the sample and varied considerably. The addition amount required by the dispersants was less than $1 / 10^{\text {th }}$ that of water to achieve similar reductions in yield stress for the SME product. 
WSRC-TR-2004-00082

Revision 0

This page intentionally left blank. 


\subsection{RECOMMENDATIONS}

Further evaluation and testing should be performed on Dolapix CE64 and Disperse-Ayd W28 to determine if implementation is possible in DWPF. The initial phase of future work will include optimization of the rheology modifier by IIT and development of a maximum concentration limit for the rheology modifiers. IIT has been commissioned to evaluate the properties of these chemicals to determine if the chemical makeup can be optimized to enhance the properties of these modifiers. An initial concentration limit based upon the DWPF flammability limit and other constraints should be calculated to determine the potential downstream impacts.

Once an optimized rheology modifier has been recommended by IIT, the second phase of future work concerning rheology modifiers should focus on the following areas:

- $\quad$ Effective $\mathrm{pH}$ range of dispersants

- Effect of boiling on dispersants

- Effect of aging on dispersants

- Impact of dispersants on foaming

- Impact of dispersants on hydrogen generation

- Impact of dispersants on glass redox

- Impact of dispersant components (such as ammonia) on process

It is anticipated that the second phase of this program will start in FY05. 
WSRC-TR-2004-00082

Revision 0

This page intentionally left blank. 
Revision 0

\section{APPENDIX A}

\section{Data From Rheological Modifier Testing}


WSRC-TR-2004-00082

Revision 0

\begin{tabular}{|c|c|c|c|c|c|c|c|c|}
\hline Sample ID & $\begin{array}{l}\text { Sample } \\
\text { Type }\end{array}$ & Additive & $\begin{array}{l}\text { Additive } \\
\text { Amount }\end{array}$ & $\begin{array}{l}\text { Active Agent } \\
\text { Concentration }\end{array}$ & $\begin{array}{c}\text { Active } \\
\text { Concentration } \\
\text { in Sample }\end{array}$ & $\begin{array}{l}\text { Yield } \\
\text { Stress }\end{array}$ & Consistency & $\begin{array}{c}\text { Yield Stress } \\
\% \text { of } \\
\text { Baseline }\end{array}$ \\
\hline & & & grams & wt \% & ppm & Pascals & $\mathrm{cP}$ & \\
\hline Rheo-12 & SRAT & None & 0 & 0 & 0 & 11.7 & 12.80 & 0.00 \\
\hline Rheo-13 & SRAT & Sodium Polyphosphate & 0.5 & 100 & 3444 & 17.0 & 14.90 & $(45.27)$ \\
\hline Rheo-14 & SRAT & Sodium Polyphosphate & 1 & 100 & 6863 & 20.7 & 16.80 & $(76.81)$ \\
\hline Rheo-15 & SME & None & 0 & 0 & 0 & 5.4 & 11.40 & 0.00 \\
\hline Rheo-16 & SME & Sodium Polyphosphate & 0.25 & 100 & 1719 & 6.3 & 13.00 & $(16.45)$ \\
\hline Rheo-17 & SME & Sodium Polyphosphate & 0.5 & 100 & 3444 & 6.2 & 12.40 & $(13.86)$ \\
\hline Rheo-18 & SME & Sodium Polyphosphate & 1 & 100 & 6863 & 6.7 & 12.50 & $(23.11)$ \\
\hline
\end{tabular}

\begin{tabular}{|c|c|c|c|c|c|c|c|c|}
\hline Sample ID & $\begin{array}{l}\text { Sample } \\
\text { Type }\end{array}$ & Additive & $\begin{array}{l}\text { Additive } \\
\text { Amount }\end{array}$ & $\begin{array}{l}\text { Active Agent } \\
\text { Concentration }\end{array}$ & $\begin{array}{c}\text { Active } \\
\text { Concentration } \\
\text { in Sample }\end{array}$ & $\begin{array}{l}\text { Yield } \\
\text { Stress }\end{array}$ & Consistency & $\begin{array}{c}\text { Yield Stress } \\
\% \text { of } \\
\text { Baseline } \\
\end{array}$ \\
\hline & & & & wt $\%$ & ppm & Pascals & $\mathrm{cP}$ & \\
\hline Rheo-45 & SRAT & None & 0.000 & 0 & 0 & 11.8 & 14.20 & 0.00 \\
\hline Rheo-46 & SME & None & 0.000 & 0 & 0 & 15.8 & 19.10 & 0.00 \\
\hline Rheo-47 & SRAT & Darvan 7 & 0.115 & 25 & 287 & 11.8 & 14.50 & $(0.42)$ \\
\hline Rheo-48 & SRAT & Darvan 7 & 0.230 & 25 & 574 & 12.4 & 14.90 & $(5.61)$ \\
\hline Rheo-49 & SME & Darvan 7 & 0.250 & 25 & 623 & 17.7 & 20.70 & $(12.30)$ \\
\hline Rheo-50 & SME & Darvan 7 & 0.500 & 25 & 1244 & 17.8 & 20.90 & $(12.87)$ \\
\hline Rheo-51 & SRAT & Duramax 3005 & 0.115 & 35 & 402 & 13.5 & 14.80 & $(14.44)$ \\
\hline Rheo-52 & SRAT & Duramax 3005 & 0.230 & 35 & 803 & 13.9 & 14.80 & $(18.18)$ \\
\hline Rheo-53 & SME & Duramax 3005 & 0.250 & 35 & 873 & 17.4 & 19.40 & $(10.27)$ \\
\hline Rheo-54 & SME & Duramax 3005 & 0.500 & 35 & 1741 & 17.8 & 19.40 & $(12.56)$ \\
\hline Rheo-55 & SRAT & Sodium Metasilicate & 0.115 & 100 & 1149 & 11.5 & 14.20 & 2.04 \\
\hline Rheo-56 & SRAT & Sodium Metasilicate & 0.230 & 100 & 2295 & 11.2 & 13.40 & 4.50 \\
\hline Rheo-57 & SME & Sodium Metasilicate & 0.250 & 100 & 2494 & 16.2 & 19.30 & $(2.73)$ \\
\hline Rheo-58 & SME & Sodium Metasilicate & 0.500 & 100 & 4975 & 15.5 & 16.50 & 1.52 \\
\hline Rheo-59 & SRAT & Dolapix CE64 & 0.115 & 65 & 747 & 10.8 & 13.30 & 8.58 \\
\hline Rheo-60 & SRAT & Dolapix CE64 & 0.230 & 65 & 1492 & 10.2 & 13.30 & 13.25 \\
\hline Rheo-61 & SME & Dolapix CE64 & 0.250 & 65 & 1621 & 16.4 & 20.10 & $(4.12)$ \\
\hline Rheo-62 & SME & Dolapix CE64 & 0.500 & 65 & 3234 & 13.8 & 18.80 & 12.68 \\
\hline
\end{tabular}


WSRC-TR-2004-00082

Revision 0

\begin{tabular}{|c|c|c|c|c|c|c|c|c|}
\hline Sample ID & $\begin{array}{l}\text { Sample } \\
\text { Type }\end{array}$ & Additive & $\begin{array}{l}\text { Additive } \\
\text { Amount } \\
\end{array}$ & $\begin{array}{l}\text { Active Agent } \\
\text { Concentration }\end{array}$ & $\begin{array}{c}\text { Active } \\
\text { Concentration } \\
\text { in Sample }\end{array}$ & $\begin{array}{l}\text { Yield } \\
\text { Stress }\end{array}$ & Consistency & $\begin{array}{c}\text { Yield Stress } \\
\% \text { of } \\
\text { Baseline } \\
\end{array}$ \\
\hline & & & wt. \% & wt $\%$ & ppm & Pascals & $\mathrm{cP}$ & \\
\hline Rheo-90 & SRAT & None & 0.0 & 0 & 0 & 3.9 & 9.20 & 0.00 \\
\hline Rheo-91 & SRAT & Sodium Metasilicate & 0.5 & 100 & 4975 & 3.9 & 8.40 & $(0.64)$ \\
\hline Rheo-92 & SRAT & Sodium Metasilicate & 1.0 & 100 & 9901 & 5.7 & 10.10 & $(47.85)$ \\
\hline Rheo-93 & SRAT & Sodium Metasilicate & 1.5 & 100 & 14778 & 5.3 & 10.30 & $(36.30)$ \\
\hline Rheo-94 & SRAT & Sodium Metasilicate & 2.0 & 100 & 19608 & 3.9 & 8.40 & 0.03 \\
\hline Rheo-95 & SRAT & Dolapix CE64 & 0.5 & 65 & 3234 & 2.5 & 8.90 & 34.73 \\
\hline Rheo-96 & SRAT & Dolapix CE64 & 1.0 & 65 & 6436 & 2.3 & 8.60 & 40.83 \\
\hline Rheo-97 & SRAT & Dolapix CE64 & 1.5 & 65 & 9606 & 2.1 & 8.60 & 45.60 \\
\hline Rheo-98 & SRAT & Dolapix CE64 & 2.0 & 65 & 12745 & 1.7 & 8.30 & 56.41 \\
\hline Rheo-99 & SRAT & Disperse-Ayd W22 & 0.5 & 30 & 1493 & 4.1 & 10.90 & $(4.30)$ \\
\hline Rheo-100 & SRAT & Disperse-Ayd W22 & 1.0 & 30 & 2970 & 3.7 & 8.10 & 5.33 \\
\hline Rheo-101 & SRAT & Disperse-Ayd W22 & 1.5 & 30 & 4433 & 2.9 & 8.80 & 25.31 \\
\hline Rheo-102 & SRAT & Disperse-Ayd W22 & 2.0 & 30 & 5882 & 2.8 & 10.70 & 27.39 \\
\hline Rheo-103 & SRAT & Disperse-Ayd W28 & 0.5 & 46 & 2289 & 1.8 & 9.00 & 52.42 \\
\hline Rheo-104 & SRAT & Disperse-Ayd W28 & 1.0 & 46 & 4554 & 1.5 & 5.20 & 62.36 \\
\hline Rheo-105 & SRAT & Disperse-Ayd W28 & 1.5 & 46 & 6798 & 0.8 & 7.50 & 78.86 \\
\hline Rheo-106 & SRAT & Disperse-Ayd W28 & 2.0 & 46 & 9020 & 0.6 & 7.60 & 84.55 \\
\hline Rheo-107 & SRAT & Sugar & 0.1 & 100 & 999 & 3.4 & 14.50 & 11.79 \\
\hline Rheo-108 & SRAT & Sugar & 0.2 & 100 & 1996 & 3.4 & 19.70 & 13.21 \\
\hline Rheo-109 & SRAT & Sugar & 0.3 & 100 & 2991 & 3.7 & 9.40 & 4.62 \\
\hline Rheo-110 & SRAT & Sugar & 0.5 & 100 & 4975 & 3.7 & 8.90 & 3.58 \\
\hline
\end{tabular}

\begin{tabular}{|c|c|c|c|c|c|c|c|c|}
\hline Sample ID & $\begin{array}{l}\text { Sample } \\
\text { Type }\end{array}$ & Additive & $\begin{array}{l}\text { Additive } \\
\text { Amount }\end{array}$ & $\begin{array}{l}\text { Active Agent } \\
\text { Concentration }\end{array}$ & $\begin{array}{c}\text { Active } \\
\text { Concentration } \\
\text { in Sample }\end{array}$ & $\begin{array}{l}\text { Yield } \\
\text { Stress }\end{array}$ & Consistency & $\begin{array}{c}\text { Yield Stress } \\
\% \text { of } \\
\text { Baseline } \\
\end{array}$ \\
\hline & & & grams & wt $\%$ & ppm & Pascals & $\mathrm{cP}$ & \\
\hline Rheo-111 & SRAT & None & 0 & 0 & 0 & 10.2 & 14.20 & 0.00 \\
\hline Rheo-112 & SRAT & Dolapix CE64 & 0.1 & 65 & 649 & 10.4 & 14.84 & $(1.27)$ \\
\hline Rheo-113 & SRAT & Dolapix CE64 & 0.25 & 65 & 1621 & 9.2 & 14.30 & 9.96 \\
\hline Rheo-114 & SRAT & Dolapix CE64 & 0.5 & 65 & 3234 & 8.0 & 14.59 & 21.91 \\
\hline Rheo-115 & SRAT & Disperse-Ayd W28 & 0.1 & 46 & 460 & 9.5 & 12.42 & 6.80 \\
\hline
\end{tabular}


WSRC-TR-2004-00082

Revision 0

\begin{tabular}{|c|c|c|c|c|c|c|c|c|}
\hline Rheo-116 & SRAT & Disperse-Ayd W28 & 0.25 & 46 & 1147 & 9.6 & 10.15 & 6.71 \\
\hline Rheo-117 & SRAT & Disperse-Ayd W28 & 0.5 & 46 & 2289 & 10.4 & 8.08 & $(1.40)$ \\
\hline Rheo-118 & SRAT & Disperse-Ayd W30 & 0.1 & 33.5 & 335 & 10.2 & 13.64 & 0.25 \\
\hline Rheo-119 & SRAT & Disperse-Ayd W30 & 0.25 & 33.5 & 835 & 9.7 & 13.10 & 4.99 \\
\hline Rheo-120 & SRAT & Disperse-Ayd W30 & 0.5 & 33.5 & 1667 & 10.3 & 13.10 & $(0.10)$ \\
\hline Rheo-121 & SRAT & Disperse-Ayd W39 & 0.1 & 44 & 440 & 10.4 & 10.15 & $(1.72)$ \\
\hline Rheo-122 & SRAT & Disperse-Ayd W39 & 0.25 & 44 & 1097 & 8.6 & 9.60 & 15.92 \\
\hline Rheo-123 & SRAT & Disperse-Ayd W39 & 0.5 & 44 & 2189 & 11.0 & 11.92 & $(7.49)$ \\
\hline Rheo-124 & SRAT & Alcosperse 149 & 0.1 & 40 & 400 & 11.0 & 1.17 & $(7.64)$ \\
\hline Rheo-125 & SRAT & Alcosperse 149 & 0.25 & 40 & 998 & 11.7 & 112.11 & $(14.28)$ \\
\hline Rheo-126 & SRAT & Alcosperse 149 & 0.5 & 40 & 1990 & 13.6 & 13.30 & $(32.49)$ \\
\hline Rheo-127 & SRAT & Alcosperse 240 & 0.1 & 44 & 440 & 13.0 & 10.20 & (27.07) \\
\hline Rheo-128 & SRAT & Alcosperse 240 & 0.25 & 44 & 1097 & 12.4 & 11.05 & $(21.21)$ \\
\hline Rheo-129 & SRAT & Alcosperse 240 & 0.5 & 44 & 2189 & 12.1 & 12.60 & $(18.11)$ \\
\hline Rheo-130 & SRAT & Alcosperse 408 & 0.1 & 41 & 410 & 10.4 & 14.81 & $(1.94)$ \\
\hline Rheo-131 & SRAT & Alcosperse 408 & 0.25 & 41 & 1022 & 10.0 & 18.22 & 2.09 \\
\hline Rheo-132 & SRAT & Alcosperse 408 & 0.5 & 41 & 2040 & 12.0 & 18.46 & $(17.09)$ \\
\hline Rheo-133 & SRAT & Alcosperse 725 & 0.1 & 35 & 350 & 11.9 & 13.52 & $(15.80)$ \\
\hline Rheo-134 & SRAT & Alcosperse 725 & 0.25 & 35 & 873 & 14.2 & 10.06 & $(38.65)$ \\
\hline Rheo-135 & SRAT & Alcosperse 725 & 0.5 & 35 & 1741 & 16.1 & 6.59 & $(57.59)$ \\
\hline Rheo-136 & SRAT & EDAPlan 470 & 0.1 & 50 & 500 & 11.1 & 13.99 & $(8.55)$ \\
\hline Rheo-137 & SRAT & EDAPlan 470 & 0.25 & 50 & 1247 & 10.9 & 14.27 & $(6.87)$ \\
\hline Rheo-138 & SRAT & EDAPlan 470 & 0.5 & 50 & 2488 & 10.9 & 14.40 & $(6.15)$ \\
\hline Rheo-139 & SRAT & EDAPlan 472 & 0.5 & 50 & 2488 & 10.4 & 13.13 & $(1.67)$ \\
\hline Rheo-140 & SRAT & EDAPlan 472 & 0.1 & 50 & 500 & 10.8 & 14.07 & $(5.67)$ \\
\hline Rheo-141 & SRAT & EDAPlan 472 & 0.25 & 50 & 1247 & 10.6 & 13.82 & $(3.99)$ \\
\hline Rheo-142 & SRAT & PomoSperse AL36 & 0.1 & 42 & 420 & 10.4 & 12.60 & $(1.27)$ \\
\hline Rheo-143 & SRAT & PomoSperse AL36 & 0.1 & 42 & 420 & 13.1 & 12.07 & $(28.25)$ \\
\hline Rheo-144 & SRAT & PomoSperse AL36 & 0.25 & 42 & 1047 & 16.7 & 10.88 & (63.38) \\
\hline
\end{tabular}


WSRC-TR-2004-00082

Revision 0

\begin{tabular}{|c|c|c|c|c|c|c|c|c|}
\hline Sample ID & $\begin{array}{l}\text { Sample } \\
\text { Type }\end{array}$ & Additive & $\begin{array}{l}\text { Additive } \\
\text { Amount }\end{array}$ & $\begin{array}{l}\text { Active Agent } \\
\text { Concentration }\end{array}$ & $\begin{array}{c}\text { Active } \\
\text { Concentration } \\
\text { in Sample }\end{array}$ & $\begin{array}{l}\text { Yield } \\
\text { Stress }\end{array}$ & Consistency & $\begin{array}{c}\text { Yield Stress } \\
\% \text { of } \\
\text { Baseline }\end{array}$ \\
\hline & & & grams & wt $\%$ & $\mathrm{ppm}$ & Pascals & $\mathrm{cP}$ & \\
\hline Rheo-145 & SME & none & 0 & 0 & 0 & 10.5 & 20.00 & 0.00 \\
\hline Rheo-146 & SME & Dolapix CE64 & 0.1 & 65 & 649 & 9.7 & 23.00 & 7.63 \\
\hline Rheo-147 & SME & Dolapix CE64 & 0.25 & 65 & 1621 & 9.7 & 23.00 & 8.00 \\
\hline Rheo-148 & SME & Dolapix CE64 & 0.5 & 65 & 3234 & 8.2 & 19.00 & 21.57 \\
\hline Rheo-149 & SME & Disperse-Ayd W28 & 0.1 & 46 & 460 & 10.8 & 20.00 & $(3.08)$ \\
\hline Rheo-150 & SME & Disperse-Ayd W28 & 0.25 & 46 & 1147 & 8.6 & 17.00 & 18.32 \\
\hline Rheo-151 & SME & Disperse-Ayd W28 & 0.5 & 46 & 2289 & 6.1 & 15.00 & 42.12 \\
\hline Rheo-152 & SME & Disperse-Ayd W30 & 0.1 & 33.5 & 335 & 11.2 & 23.00 & $(6.29)$ \\
\hline Rheo-153 & SME & Disperse-Ayd W30 & 0.25 & 33.5 & 835 & 10.7 & 19.00 & $(1.43)$ \\
\hline Rheo-154 & SME & Disperse-Ayd W30 & 0.5 & 33.5 & 1667 & 10.9 & 19.00 & (3.33) \\
\hline Rheo-155 & SME & Disperse-Ayd W39 & 0.1 & 44 & 440 & 10.5 & 21.00 & $(0.10)$ \\
\hline Rheo-156 & SME & Disperse-Ayd W39 & 0.25 & 44 & 1097 & 9.7 & 19.00 & 7.52 \\
\hline Rheo-157 & SME & Disperse-Ayd W39 & 0.5 & 44 & 2189 & 10.5 & 18.00 & 0.29 \\
\hline Rheo-158 & SME & Alcosperse 149 & 0.1 & 40 & 400 & 12.7 & 24.15 & $(20.57)$ \\
\hline Rheo-159 & SME & Alcosperse 149 & 0.25 & 40 & 998 & 13.1 & 23.89 & $(24.67)$ \\
\hline Rheo-160 & SME & Alcosperse 149 & 0.5 & 40 & 1990 & 12.5 & 23.99 & $(18.57)$ \\
\hline Rheo-161 & SME & Alcosperse 240 & 0.1 & 44 & 440 & 13.2 & 21.00 & $(25.33)$ \\
\hline Rheo-162 & SME & Alcosperse 240 & 0.25 & 44 & 1097 & 17.8 & 15.00 & $(69.14)$ \\
\hline Rheo-163 & SME & Alcosperse 240 & 0.5 & 44 & 2189 & 18.4 & 14.00 & $(74.76)$ \\
\hline Rheo-164 & SME & Alcosperse 408 & 0.1 & 41 & 410 & 11.9 & 23.00 & $(13.62)$ \\
\hline Rheo-165 & SME & Alcosperse 408 & 0.25 & 41 & 1022 & 14.2 & 20.00 & $(35.62)$ \\
\hline Rheo-166 & SME & Alcosperse 408 & 0.5 & 41 & 2040 & 16.0 & 23.00 & $(52.38)$ \\
\hline Rheo-167 & SME & Alcosperse 725 & 0.1 & 35 & 350 & 12.2 & 230.00 & $(16.38)$ \\
\hline Rheo-168 & SME & Alcosperse 725 & 0.25 & 35 & 873 & 13.0 & 24.11 & $(23.52)$ \\
\hline Rheo-169 & SME & Alcosperse 725 & 0.5 & 35 & 1741 & 11.5 & 25.36 & $(9.81)$ \\
\hline Rheo-170 & SME & EDAPlan 470 & 0.1 & 50 & 500 & 11.7 & 24.00 & $(10.95)$ \\
\hline Rheo-171 & SME & EDAPlan 470 & 0.25 & 50 & 1247 & 11.4 & 21.00 & $(8.76)$ \\
\hline Rheo-172 & SME & EDAPlan 470 & 0.5 & 50 & 2488 & 12.6 & 23.00 & $(19.71)$ \\
\hline Rheo-173 & SME & EDAPlan 472 & 0.1 & 50 & 500 & 11.9 & 22.00 & $(13.24)$ \\
\hline Rheo-174 & SME & EDAPlan 472 & 0.25 & 50 & 1247 & 11.5 & 24.00 & $(9.71)$ \\
\hline
\end{tabular}


WSRC-TR-2004-00082

Revision 0

\begin{tabular}{|c|c|c|c|c|c|c|c|c|}
\hline Rheo-175 & SME & EDAPlan 472 & 0.5 & 50 & 2488 & 12.1 & 22.00 & (14.86) \\
\hline Rheo-176 & SME & PomoSperse AL36 & 0.1 & 42 & 420 & 11.8 & 22.00 & (12.48) \\
\hline Rheo-177 & SME & PomoSperse AL36 & 0.25 & 42 & 1047 & 15.3 & 20.00 & $(46.10)$ \\
\hline Rheo-178 & SME & PomoSperse AL36 & 0.5 & 42 & 2090 & 18.1 & 18.00 & (72.29) \\
\hline Rheo-179 & SME & Water & 10 & 100 & 90,909 & 6.0 & 17.00 & 43.05 \\
\hline Rheo-180 & SME & Water & 20 & 100 & 166,667 & 4.0 & 13.00 & 62.38 \\
\hline Rheo-181 & SME & Water & 30 & 100 & 230,769 & 2.6 & 11.00 & 75.05 \\
\hline Rheo-182 & SME & Water & 40 & 100 & 285,714 & 1.8 & 10.00 & 82.57 \\
\hline
\end{tabular}

\begin{tabular}{|c|c|c|c|c|c|c|c|c|}
\hline Sample ID & $\begin{array}{l}\text { Sample } \\
\text { Type }\end{array}$ & Additive & $\begin{array}{l}\text { Additive } \\
\text { Amount }\end{array}$ & $\begin{array}{l}\text { Active Agent } \\
\text { Concentration }\end{array}$ & $\begin{array}{c}\text { Active } \\
\text { Concentration } \\
\text { in Sample }\end{array}$ & $\begin{array}{l}\text { Yield } \\
\text { Stress }\end{array}$ & Consistency & $\begin{array}{c}\text { Yield Stress } \\
\% \text { of } \\
\text { Baseline } \\
\end{array}$ \\
\hline & & & grams & wt \% & ppm & Pascals & $\mathrm{cP}$ & \\
\hline Rheo-183 & SME & Cyanamer P-35 & 0.10 & 50 & 500 & 17.0 & 20.00 & $(62.29)$ \\
\hline Rheo-184 & SME & Cyanamer P-35 & 0.25 & 50 & 1247 & 27.9 & 3.89 & $(165.71)$ \\
\hline Rheo-185 & SME & Cyanamer P-35 & 0.50 & 50 & 2488 & 33.5 & -4.70 & $(219.43)$ \\
\hline Rheo-186 & SME & Cyanamer P-70 & 0.10 & 49.8 & 498 & 13.4 & 24.48 & $(27.24)$ \\
\hline Rheo-187 & SME & Cyanamer P-70 & 0.25 & 49.8 & 1242 & 13.7 & 21.64 & $(30.48)$ \\
\hline Rheo-188 & SME & Cyanamer P-70 & 0.50 & 49.8 & 2478 & 11.4 & 20.62 & $(8.76)$ \\
\hline Rheo-189 & SRAT & Cyanamer P-35 & 0.10 & 50 & 500 & 12.8 & 14.51 & $(24.51)$ \\
\hline Rheo-190 & SRAT & Cyanamer P-35 & 0.25 & 50 & 1247 & 21.6 & 9.43 & $(110.45)$ \\
\hline Rheo-191 & SRAT & Cyanamer P-35 & 0.50 & 50 & 2488 & 37.8 & -9.94 & (269.43) \\
\hline Rheo-192 & SRAT & Cyanamer P-70 & 0.10 & 49.8 & 498 & 10.8 & 13.98 & $(5.37)$ \\
\hline Rheo-193 & SRAT & Cyanamer P-70 & 0.25 & 49.8 & 1242 & 11.3 & 14.03 & $(9.86)$ \\
\hline Rheo-194 & SRAT & Cyanamer P-70 & 0.50 & 49.8 & 2478 & 11.3 & 13.21 & $(10.25)$ \\
\hline
\end{tabular}

